\title{
Utility of arterial spin labeling perfusion magnetic resonance imaging in prediction of angiographic vascularity of meningiomas
}

\author{
Roh-Eul Yoo, MD,,2 Tae Jin Yun, MD,, ${ }^{1,2}$ Young Dae Cho, MD, ${ }^{1,2}$ Jung Hyo Rhim, MD, ${ }^{1}$ \\ Koung Mi Kang, MD, ${ }^{1,2}$ Seung Hong Choi, MD, ${ }^{1-3}$ Ji-hoon Kim, MD, ${ }^{1,2}$ Jeong Eun Kim, MD, ${ }^{4}$ \\ Hyun-Seung Kang, MD, PhD, ${ }^{4}$ Chul-Ho Sohn, MD, ${ }^{1-3}$ Sun-Won Park, MD, ${ }^{2,5}$ and \\ Moon Hee Han, MD1-3
}

\begin{abstract}
Departments of ${ }^{1}$ Radiology and ${ }^{4}$ Neurosurgery, Seoul National University Hospital; ${ }^{2}$ Department of Radiology, Seoul National University College of Medicine; ${ }^{3}$ nstitute of Radiation Medicine, Seoul National University Medical Research Center; and ${ }^{5}$ Department of Radiology, Boramae Medical Center, Seoul, Korea
\end{abstract}

OBJECTIVE Arterial spin labeling perfusion-weighted imaging (ASL-PWI) enables quantification of tissue perfusion without contrast media administration. The aim of this study was to explore whether cerebral blood flow (CBF) from ASLPWI can reliably predict angiographic vascularity of meningiomas.

METHODS Twenty-seven patients with intracranial meningiomas, who had undergone preoperative ASL-PWI and digital subtraction angiography prior to resection, were included. Angiographic vascularity was assessed using a 4-point grading scale and meningiomas were classified into 2 groups: low vascularity (Grades 0 and $1 ; n=11$ ) and high vascularity (Grades 2 and $3 ; n=16$ ). Absolute CBF, measured at the largest section of the tumor, was normalized to the contralateral gray matter. Correlation between the mean normalized $\mathrm{CBF}(\mathrm{nCBF})$ and angiographic vascularity was determined and the mean $\mathrm{nCBF}$ values of the 2 groups were compared. Diagnostic performance of the $\mathrm{nCBF}$ for differentiating between the 2 groups was assessed.

RESULTS The $n C B F$ had a significant positive correlation with angiographic vascularity $(\rho=0.718 ; p<0.001)$. The high-vascularity group had a significantly higher $\mathrm{nCBF}$ than the low-vascularity group $(3.334 \pm 2.768$ and $0.909 \pm 0.468$, respectively; $p=0.003$ ). At the optimal $n C B F$ cutoff value of 1.733 , sensitivity and specificity for the differential diagnosis of the 2 groups were $69 \%(95 \% \mathrm{Cl} 41 \%-89 \%)$ and $100 \%(95 \% \mathrm{Cl} 72 \%-100 \%)$, respectively. The area under the receiver operating characteristic curve was $0.875(p<0.001)$.

CONCLUSIONS ASL-PWI may provide a reliable and noninvasive means of predicting angiographic vascularity of meningiomas. It may thus assist in selecting potential candidates for preoperative digital subtraction angiography and embolization in clinical practice.

http://thejns.org/doi/abs/10.3171/2015.8.JNS151211

KEY WORDS arterial spin labeling; perfusion magnetic resonance imaging; angiographic vascularity; meningiomas; oncology

$\mathrm{M}$ ENINGIOMAS are the most frequently diagnosed primary brain tumors. ${ }^{20}$ They are hypervascular tumors commonly supplied by dural arteries such as the middle meningeal, accessory meningeal, ascending pharyngeal, and occipital transmastoid perforating branches of the external carotid artery (ECA). Arterial feeders can also arise from tentorial and inferolateral trunk branches of the internal carotid artery (ICA), the posterior meningeal branch of the vertebral artery, and pial branches. ${ }^{7}$

Preoperative embolization is considered as an adjunctive treatment for meningiomas in selected cases to decrease the technical difficulty of resection and maximize the rate of complete resection, given the hypervascular nature of the tumor. ${ }^{13}$ Furthermore, information obtained from diagnostic angiography itself, including tumor vas-

ABBREVIATIONS ASL-PWI = arterial spin labeling perfusion-weighted imaging; $\mathrm{CBF}=$ cerebral blood flow; $\mathrm{DSA}=$ digital subtraction angiography; $\mathrm{ECA}=$ external carotid artery; ICA = internal carotid artery; $\mathrm{nCBF}=$ normalized $\mathrm{CBF} ; \mathrm{ROC}$ = receiver operating characteristic.

SUBMITTED May 24, 2015. ACCEPTED August 17, 2015.

INCLUDE WHEN CITING Published online January 29, 2016; DOI: 10.3171/2015.8.JNS151211. 
cularity or vascular anatomy of feeding arteries and draining veins, can also help surgical planning. ${ }^{17}$ Nonetheless, digital subtraction angiography (DSA) is an invasive imaging modality, with reported neurological complication rates ranging from $0.34 \%$ to $1.3 \%$.,16,21

More recently, normalized cerebral blood volume derived from dynamic susceptibility contrast-enhanced perfusion-weighted imaging (PWI) has been found to correlate well with angiographic vascularity of meningiomas and to aid in the differential diagnosis of low-vascularity from high-vascularity meningiomas. ${ }^{17}$ Over the past decade, cerebral blood flow (CBF), determined from arterial spin labeling (ASL)-PWI, has been shown to have a strong association with perfusion parameters obtained from dynamic susceptibility contrast-enhanced PWI in several studies. ${ }^{3,8-10,18,19,23}$ ASL, unlike dynamic susceptibility contrast-enhanced PWI, is a noninvasive perfusion MR technique that uses magnetically labeled water as a diffusible tracer in place of an exogenous contrast material, such as a gadolinium-based agent, to measure $\mathrm{CBF}$ values. ${ }^{1,5,6}$

To our knowledge, however, the correlation between ASL-PWI and angiographic vascularity of meningiomas has not been elucidated. Therefore, the purpose of the present study was to explore whether CBF from ASL-PWI can reliably predict angiographic vascularity of meningiomas.

\section{Methods}

This retrospective study was approved by our institutional review board, and informed consent was waived.

\section{Patient Selection}

Two hundred eighty-three patients with intracranial meningiomas who had undergone preoperative DSA prior to resection at our institution between January 2012 and October 2014 were selected from our radiology report database. Two hundred fifty-five patients were excluded for having had no preoperative ASL-PWI, and 1 patient was excluded due to a recent history of radiation therapy. A total of 27 consecutive patients were included in this study.

\section{Image Acquisition}

All patients underwent MRI with a 1.5-T (Signa HDxt; GE Healthcare $[n=18]$ ) or a 3-T (Verio; Siemens $[n=5]$; Discovery 750; GE Healthcare [ $\mathrm{n}=4]$ ) MR scanner using an 8- or 32-channel head coil.

The imaging protocol included axial fast spin-echo T2weighted, axial spin-echo T1-weighted, contrast-enhanced axial spin-echo T1-weighted sequences, and ASL-PWI. For the contrast-enhanced T1-weighted sequence, axial T1-weighted sequence was repeated after the intravenous administration of a single dose of $0.1 \mathrm{mmol} / \mathrm{kg}$ body weight of gadobutrol (Gadavist; Bayer Schering Pharma AG). A fat-suppression pulse was applied to the axial T1weighted imaging after contrast administration.

Arterial spin labeling was performed using either a pseudocontinuous $(n=22)$ or pulsed $(n=5)$ labeling method. The label duration and postlabel delay were 1.5 seconds and 1.5 seconds for the pseudocontinuous ASL and $700 \mathrm{msec}$ and $1100 \mathrm{msec}$ for the pulsed ASL, respectively. A quantitative perfusion map of $\mathrm{CBF}$ was obtained afterward by fitting the signal intensity change between the labeled and control images to a previously published model. ${ }^{24}$ Specific imaging parameters for all sequences are provided in Table 1 .

\section{Image Analysis}

Visual Assessment of Angiographic Vascularity on DSA

A neurointerventionist (Y.D.C., with 10 years of experience) and a neuroradiologist (T.J.Y., with 12 years of experience), who were blinded to ASL-PWI findings, reviewed the entire series of angiographic images and graded the degree of angiographic vascularity on the basis of previously reported criteria: Grade 0, none; Grade 1, minimal; Grade 2, moderate; and Grade 3, marked. ${ }^{17}$ Interobserver differences were resolved by consensus. In cases of multiple feeders, the vascularity of the tumor as a whole was estimated by summing tumor stains observed in each selective angiography.

Meningiomas were then further classified into 2 groups, according to the angiographic vascularity grade: low-vascularity meningiomas (Grades 0 and 1), for which preoperative embolization or even DSA is considered unnecessary; and high-vascularity meningiomas (Grades 2 and 3), which are potential candidates for embolization. ${ }^{17}$

\section{Quantitative Analysis of CBF on ASL-PWI}

On a picture archiving and communication system workstation, regions of interest that contained the entire contrast-enhancing areas, excluding the areas of necrosis or cysts, were drawn in the most representative (i.e., largest) sections of each CBF map by 1 neuroradiologist (R.E.Y., with 5 years of experience) to determine the absolute $\mathrm{CBF}$ of the tumor $\left(\mathrm{CBF}_{\text {tumor }}\right)$. At the same time, to adjust for the interindividual variation among MR scanners and basal CBF, additional regions of interest were drawn within the gray matter of normal contralateral parenchyma at the same slice levels for $\mathrm{CBF}$ normalization. Subsequently, normalized $\mathrm{CBF}$ (nCBF) was calculated as $\mathrm{nCBF}=\mathrm{CBF}_{\text {tumor }} / \mathrm{CBF}_{\text {gray matter }}$.

\section{Statistical Analysis}

All statistical analyses were performed using MedCalc software (version 11.1.1.0). For each parameter, normality of the data was assessed with the Kolmogorov-Smirnov test. Results with $\mathrm{p}$ values less than 0.05 were considered statistically significant.

The nCBF values of the 4 different grades of angiographic vascularity were compared using the JonckheereTerpstra trend test of independent samples with multiple pairwise comparisons. The Spearman correlation coefficient was calculated to assess correlation between the $\mathrm{nCBF}$ and angiographic vascularity of meningiomas.

The unpaired Student t-test or Fisher exact test was used to compare clinical and imaging variables of the lowand high-vascularity groups, including age and sex of the patients as well as the mean size and $\mathrm{nCBF}$ of the tumors.

Furthermore, a receiver operating characteristic (ROC) curve was constructed for the nCBF to determine a cutoff level that provided a balance between sensitivity and spec- 
TABLE 1. Parameters for MRI studies

\begin{tabular}{|c|c|c|c|c|}
\hline \multirow[b]{2}{*}{ Parameter } & \multirow{2}{*}{$\begin{array}{l}\text { Axial Spin-Echo T1- } \\
\text { Weighted Sequence }\end{array}$} & \multirow{2}{*}{$\begin{array}{l}\text { Axial Fast Spin-Echo } \\
\text { T2-Weighted Sequence }\end{array}$} & \multicolumn{2}{|c|}{ ASL-PWI } \\
\hline & & & Pseudocontinuous* & Pulsedt \\
\hline Repetition time (msec) & $467-567$ & $4850-5330$ & $4446-4564$ & 3000 \\
\hline Echo time (msec) & $8-9$ & $92-127$ & $9.4-9.9$ & 12 \\
\hline Echo train length & 1 & $16-28$ & 1 & 37 \\
\hline Flip angle $\left(^{\circ}\right)$ & 90 & $90-142$ & $111-155$ & 90 \\
\hline Section thickness (mm) & 5 & 5 & $5-6$ & 6 \\
\hline Intersection gap (mm) & 1 & 1 & 0 & 1.5 \\
\hline Field of view (mm) & $220 \times 220$ & $220 \times 220$ & $240 \times 240$ & $240 \times 210$ \\
\hline Matrix & $320 \times 192$ & $448 \times 256$ & $128 \times 128$ & $64 \times 49$ \\
\hline No. of signals acquired & 1 & 2 & $2-3$ & 1 \\
\hline No. of sections & 26 & 26 & $32-44$ & 14 \\
\hline
\end{tabular}

ificity for the differential diagnosis between the 2 groups. The sensitivity and specificity at the optimal cutoff level, as well as the area under the ROC curve, were calculated with 95\% CIs. In addition, results from the ROC analysis were tested by a leave-one-out cross-validation method.

Finally, the difference in the $\mathrm{nCBF}$ between the group with embolization and the group with DSA only was evaluated using the Mann-Whitney U test.

\section{Results}

\section{Patient Ages and Tumor Characteristics}

There were 12 men (mean age 64 years, range 33-78 years) and 15 women (mean age 53 years, range 32-63 years). The mean tumor size was $5.19 \pm 1.63 \mathrm{~cm}$. The histological subtypes comprised 2 angiomatous and 25 nonangiomatous (i.e., 9 meningothelial, 2 transitional, 3 fibroblastic, 1 sclerosing, 1 secretory, 1 microcystic, 6 atypical, and 2 anaplastic) meningiomas.

\section{Difference in nCBF Values According to Angiographic Vascularity}

Interobserver agreement was substantial $(\kappa=0.723)$ for grading of angiographic vascularity. The angiographic vascularity on consensus readings was Grade 0 in 1, Grade 1 in 10, Grade 2 in 13, and Grade 3 in 3 meningiomas.

The $\mathrm{nCBF}$ increased with higher angiographic vascularity grade, as shown in Table 2 and Fig. 1 . The Jonckheere-Terpstra trend test showed a statistically significant difference in the $\mathrm{nCBF}$ across the 4 angiographic vascularity grades $(\mathrm{p}<0.001)$. In pairwise comparisons, significant differences in the nCBF were observed between any 2 grades of angiographic vascularity $(\mathrm{p}<0.05)$, except between Grades 0 and 1. Moreover, there was a significant positive correlation between the $\mathrm{nCBF}$ and angiographic vascularity $(\rho=0.718 ; p<0.001)$.

Subgroup analysis according to the magnetic field strengths showed a significant positive correlation between the $\mathrm{nCBF}$ and angiographic vascularity at both $1.5 \mathrm{~T}(\rho=$ $0.753 ; \mathrm{p}<0.001)$ and $3 \mathrm{~T}(\rho=0.721 ; \mathrm{p}=0.028)$. In addition, the $\mathrm{nCBF}$ was significantly higher in the high-vascularity group $(n=16)$ than in the low-vascularity group $(n$
$=11)($ mean $\pm \mathrm{SD}, 3.334 \pm 2.768$ [values are reported as mean \pm SD unless otherwise specified $]$ and $0.909 \pm 0.468$, respectively; $\mathrm{p}=0.003$ ) (Fig. 1).

\section{Diagnostic Performance of the $\mathrm{nCBF}$ in Predicting Angiographic Vascularity}

At the optimal nCBF cutoff value of 1.733 , sensitivity and specificity for the differential diagnosis of the lowvascularity from high-vascularity meningiomas were $69 \%$ (11 of 16 patients; $95 \%$ CI $41 \%-89 \%$ ) and $100 \%$ (11 of 11 patients; 95\% CI 72\%-100\%), respectively. The area under the ROC curve was 0.875 (95\% CI 0.691-0.970; $\mathrm{p}<$ 0.001) (Table 3). Leave-one-out cross-validation showed a sensitivity of $69 \%$ and a specificity of $100 \%$, which were identical to the primary results. Tumor size, patient age, and sex of patients did not significantly differ between the 2 groups $(\mathrm{p}=0.980, \mathrm{p}=0.103$, and $\mathrm{p}=1.000$, respectively) (Table 4).

The majority of the patients (12 of 16 [75\%]) in the high-vascularity group underwent preoperative embolization, whereas the majority of the patients (8 of 11 [73\%]) in the low-vascularity group underwent DSA only. Despite angiographic findings suggestive of high vascularity, embolization was not performed in 3 patients due to small size of arterial feeders and in 1 patient due to ICA origin of the main feeder. On the other hand, embolization was performed in 3 patients with low-vascularity meningiomas due to the treating interventionist's preference or the relative ease of microcatheter navigation. Initially, no signifi-

TABLE 2. Comparison of $n C B F$ values of the 4 angiographic vascularity grades

\begin{tabular}{ccl}
\hline Grade & No. of Patients & \multicolumn{1}{c}{ nCBF $^{*}$} \\
\hline 0 & 1 & 0.493 \\
\hline 1 & 10 & $0.951 \pm 0.471(0.911,0.595-1.193)$ \\
\hline 2 & 13 & $2.604 \pm 2.227(1.765,1.079-2.927)$ \\
\hline 3 & 3 & $6.494 \pm 3.045(7.250,5.196-8.170)$ \\
\hline
\end{tabular}

* Data are expressed as the mean \pm SD. Data in the parentheses are the median, 1st-3rd quartiles. The $\mathrm{nCBF}$ represents the CBF of the tumor normalized to the contralateral gray matter. 

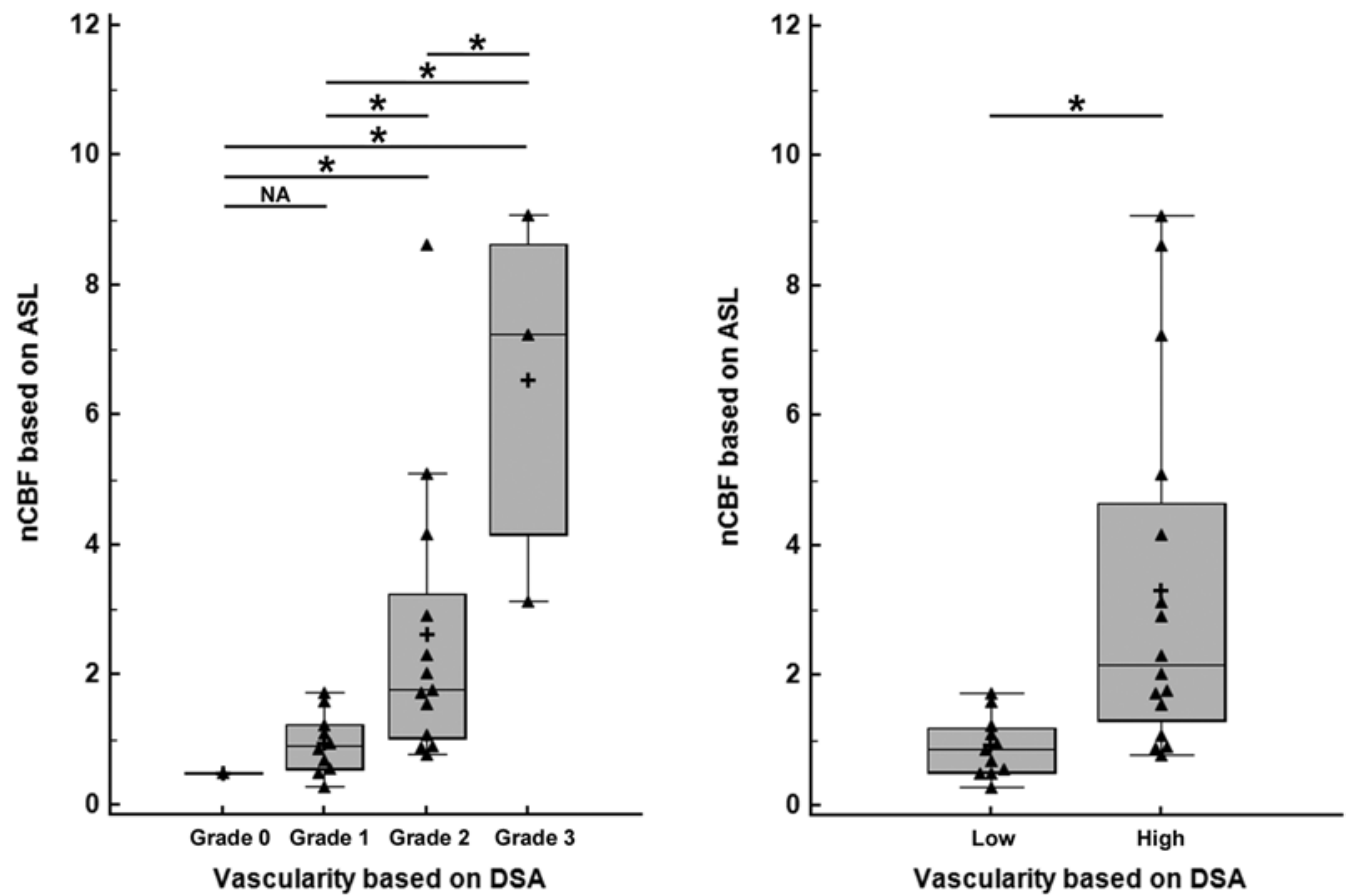

FIG. 1. Box-and-whisker displays of the $\mathrm{nCBF}$ for the 4 angiographic vascularity grades. Left: There was a significant difference in the nCBF when comparing any 2 grades of angiographic vascularity $(p<0.05)$, except between Grades 0 and 1 . Right: The high-vascularity group (Grades 2 and 3 ) had a significantly higher nCBF than the low-vascularity group (Grades 0 and 1$)$ (3.334 \pm 2.768 and $0.909 \pm 0.468$, respectively; $p=0.003$ ). There was a significant positive correlation between the $\mathrm{nCBF}$ and angiographic vascularity $(\rho=0.718 ; p<0.001)$. The line across the boxes denotes the median value, whereas the ends of the boxes represent the first and third quartiles. The ends of each plot indicate the smallest and largest values, and cases beyond this range are displayed individually. $\mathrm{NA}=$ not available; $+=$ mean value. ${ }^{*} p<0.05$.

cant difference in the $\mathrm{nCBF}$ was found between the group with embolization and the group with DSA only $(2.218 \pm$ 2.272 and $2.506 \pm 2.741$, respectively; $\mathrm{p}=0.922$ ). However, the nCBF of the group with embolization significantly differed from that of the group with DSA only, when the exceptional cases were excluded from the analysis $(2.618$ \pm 2.384 and $1.000 \pm 0.481$, respectively; $\mathrm{p}=0.025$ ). At the optimal nCBF cutoff value of 1.733 , sensitivity and specificity for the differential diagnosis between the 2 groups were $58 \%$ (7 of 12 patients; 95\% CI 28\%-85\%) and $100 \%$ ( 8 of 8 patients; 95\% CI 63\%-100\%), respectively. The area under the ROC curve was 0.802 (95\% CI 0.566-0.944; $\mathrm{p}=0.003$ ) (Table 3).

\section{Estimated Blood Loss and Postoperative Complications}

Three of the 16 patients with high-vascularity meningiomas experienced postoperative complications (neurological complication [ $\mathrm{n}=1]$, epidural hematoma $[\mathrm{n}=1]$, and intracerebral hemorrhage and neurological complication $[n=1])$, despite preoperative embolization in 1 of these 3 patients. In comparison, 1 of the 11 patients with low-vascularity meningiomas had a complication (intraventricular hemorrhage). In addition, among the 12 patients who underwent DSA only, the mean estimated blood loss was $970 \pm 449 \mathrm{ml}$ for those with high-vascularity $(\mathrm{n}=4)$ and $867 \pm 718 \mathrm{ml}$ for those with low-vascularity meningiomas $(n=8)$. Statistically significant differences, however, were not found in the study population. Representative images, including ASL-PWI and DSA in patients with meningiomas, are shown in Figs. 2-4.

\section{Discussion}

The results of our study suggest that angiographic vascularity of meningiomas can be reliably predicted using the $\mathrm{nCBF}$ from ASL-PWI, a noninvasive perfusion MR technique. The $\mathrm{nCBF}$ had a significant positive correlation

TABLE 3. Diagnostic performance of the $\mathrm{nCBF}$ in predicting angiographic vascularity

\begin{tabular}{lccccc}
\hline \multicolumn{1}{c}{ Parameter } & Area Under the ROC Curve $(95 \% \mathrm{Cl})$ & ${\text { Sensitivity }(95 \% \mathrm{Cl})^{*}}$ & Specificity $\left(95 \%\right.$ Cl) $^{*}$ & Cutoff Value & $\mathrm{p} \mathrm{Value}$ \\
\hline Angiographic vascularity & $0.875(0.691-0.970)$ & $69 \%(41 \%-89 \%)$ & $100 \%(72 \%-100 \%)$ & 1.733 & $<0.001$ \\
\hline Preop embolization & $0.802(0.566-0.944)$ & $58 \%(28 \%-85 \%)$ & $100 \%(63 \%-100 \%)$ & 1.733 & 0.003 \\
\hline
\end{tabular}

* Sensitivities and specificities for differentiating the low-vascularity from the high-vascularity meningiomas and the group with embolization from the group with DSA only.

$\dagger$ Exceptional cases (i.e., high-vascularity meningiomas in which embolization was not performed due to the small size of arterial feeders [ $\mathrm{n}=3$ ] or ICA origins of arterial feeders $[n=1]$, and low-vascularity meningiomas in which embolization was performed due to the treating interventionist's preference or the relative ease of microcatheter navigation $[n=3]$ ) were excluded from the analysis. 
TABLE 4. Clinical and imaging variables of the low- and high-vascularity groups

\begin{tabular}{cccc}
\hline Variable & $\begin{array}{c}\text { Low-Vascularity } \\
\text { Meningiomas* }\end{array}$ & $\begin{array}{c}\text { High-Vascularity } \\
\text { Meningiomas* }\end{array}$ & p Value \\
\hline Sex & & & 1.000 \\
\hline$M$ & 5 patients & 7 patients & \\
\hline F & 6 patients & 9 patients & \\
\hline Age $(y r s)$ & $61.91 \pm 10.41$ & $54.13 \pm 12.54$ & 0.103 \\
\hline Size $(\mathrm{cm})$ & $5.24 \pm 1.60$ & $5.22 \pm 1.81$ & 0.980 \\
\hline nCBF† & $0.909 \pm 0.468$ & $3.334 \pm 2.768$ & 0.003 \\
\hline
\end{tabular}

* Unless otherwise indicated, data are expressed as the mean \pm SD.

$\dagger$ The $\mathrm{nCBF}$ represents the CBF of the tumor normalized to the contralateral gray matter.

with the angiographic vascularity grade of meningiomas. Furthermore, the nCBF was significantly higher in the high-vascularity group than in the low-vascularity group, and the diagnostic performance of the $\mathrm{nCBF}$ in differentiating between the 2 groups was high.

During the past decade, there has been growing interest in ASL-PWI for its potential to enable consecutive and absolute quantification of tissue perfusion without contrast media administration, unlike dynamic susceptibility contrast-enhanced PWI. ${ }^{12,14}$ The lack of need for contrast agents is considered particularly beneficial for patients with impaired renal function, in whom the risk of developing nephrogenic systemic fibrosis after contrast administration is higher.

The utility of ASL-PWI in the evaluation of brain tumors has been explored in a number of recent clinical studies. $3,8,12,18,19,22$ For meningiomas, the relative CBF calculated from continuous ASL imaging was found to have a significant positive correlation with microvessel area and thus to facilitate the differential diagnosis between the angiomatous subtype and others. ${ }^{9}$ Similarly, in a recent study by Koizumi et al., the absolute CBF from pseudocontinuous ASL-PWI, rather than the relative CBF, was used to reflect the tumor vascularity. In their study, both the mean and maximum absolute $\mathrm{CBF}$ values were shown to have significant positive correlations with microvascular density. ${ }^{11}$

In this study, we investigated whether the $\mathrm{nCBF}$ from pseudocontinuous ASL maps could serve as a reliable imaging biomarker for angiographic vascularity of meningiomas. Our study demonstrated a significant positive correlation between the $\mathrm{nCBF}$ and angiographic vascularity, in keeping with the finding of the previous study, in which normalized cerebral blood volume obtained from dynamic susceptibility contrast-enhanced PWI was used. ${ }^{17}$

Conventional angiography can provide some valuable information that may help in planning surgery. However, a non-negligible neurological complication rate of up to $1.3 \%$ has been previously reported, suggesting that special care needs to be taken in patient selection. ${ }^{4,16,21}$ In general, invasive angiographic evaluation is considered to be justified when the anticipated benefit exceeds the procedural risk for neurological complications. ${ }^{16}$ The anticipated benefit is presumed to be maximal in those patients with angiographic findings that prompt preoperative embolization. Several studies have addressed the potential benefits of embolization. Borg et al. found that complete devascularization could lower blood transfusion requirements. ${ }^{2}$ Another study by Raper et al. also concluded that
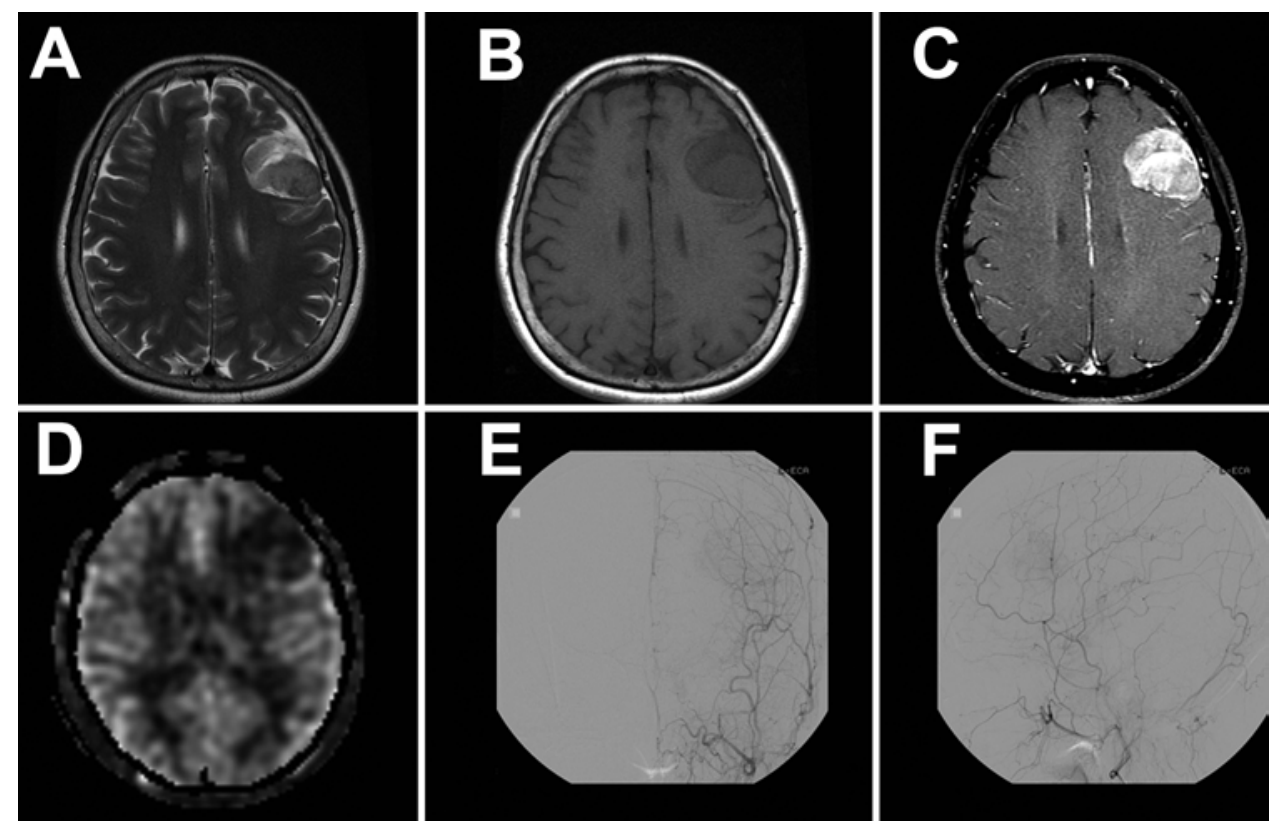

FIG. 2. Low-vascularity meningioma in a 60-year-old woman. Axial T2- (A) and T1-weighted (B and C) images demonstrate a $\mathrm{T} 2$ iso- to hyperintense, dural-based mass with relatively strong enhancement at the left frontal convexity. On pseudocontinuous ASL-PWI (D) acquired at 1.5 T, CBF elevation is not apparent in the mass ( $\mathrm{nCBF}=0.286$ ). Selective DSA of the ECA (anteroposterior $[\mathrm{E}]$ and lateral $[\mathrm{F}]$ projections) revealed Grade 1 angiographic vascularity with a tumor supply from frontal branches of the left middle meningeal artery. 

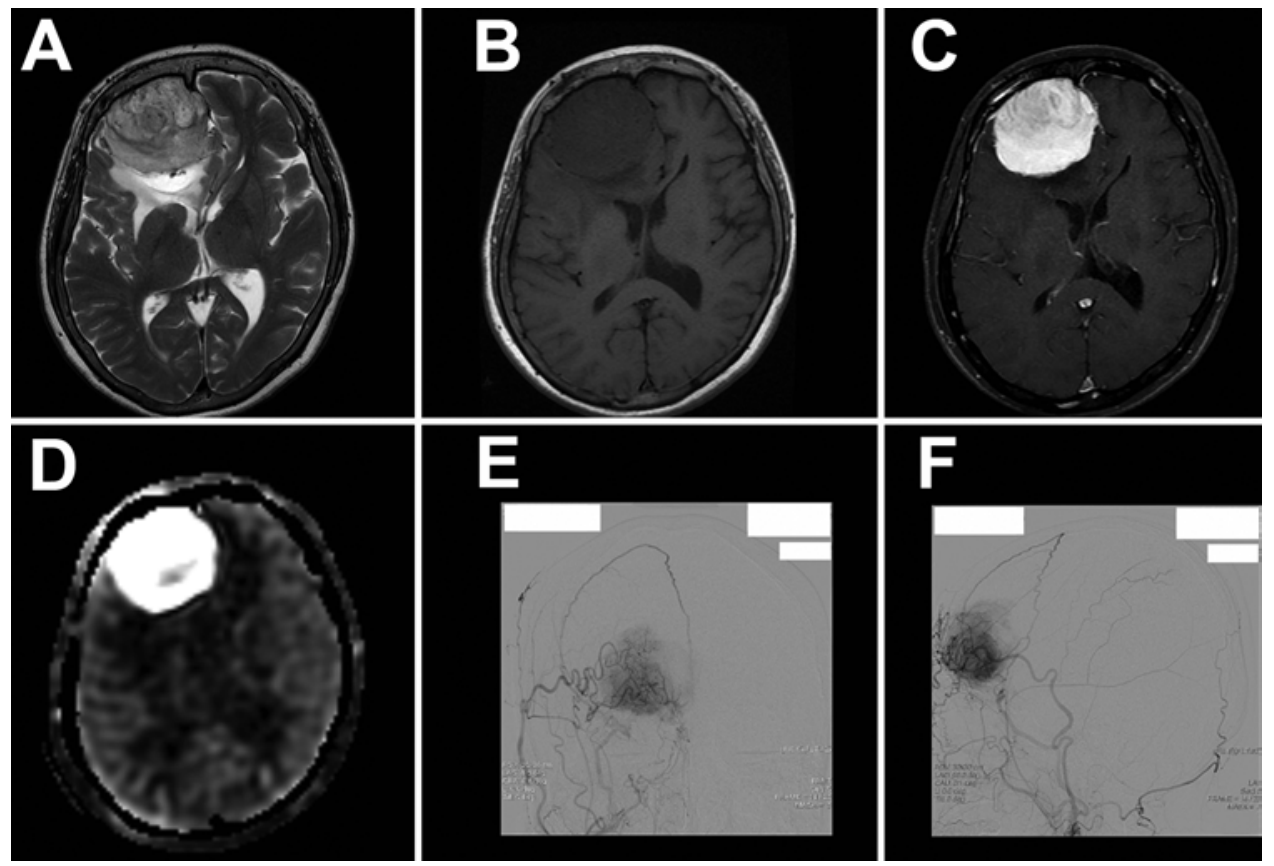

FIG. 3. High-vascularity meningioma in a 61-year-old woman. Axial T2- (A) and T1-weighted (B and C) images depict a T2 iso- to hyperintense, dural-based mass with homogeneous strong enhancement at the right frontal convexity. The mass shows a markedly increased perfusion (nCBF $=9.089$ ) on pseudocontinuous ASL-PWI (D) acquired at 1.5 T. Selective DSA of the ECA (anteroposterior [E] and lateral [F] projections) revealed a hypervascular mass with Grade 3 angiographic vascularity, which prompted preoperative embolization. The superficial temporal artery was the main arterial feeder.
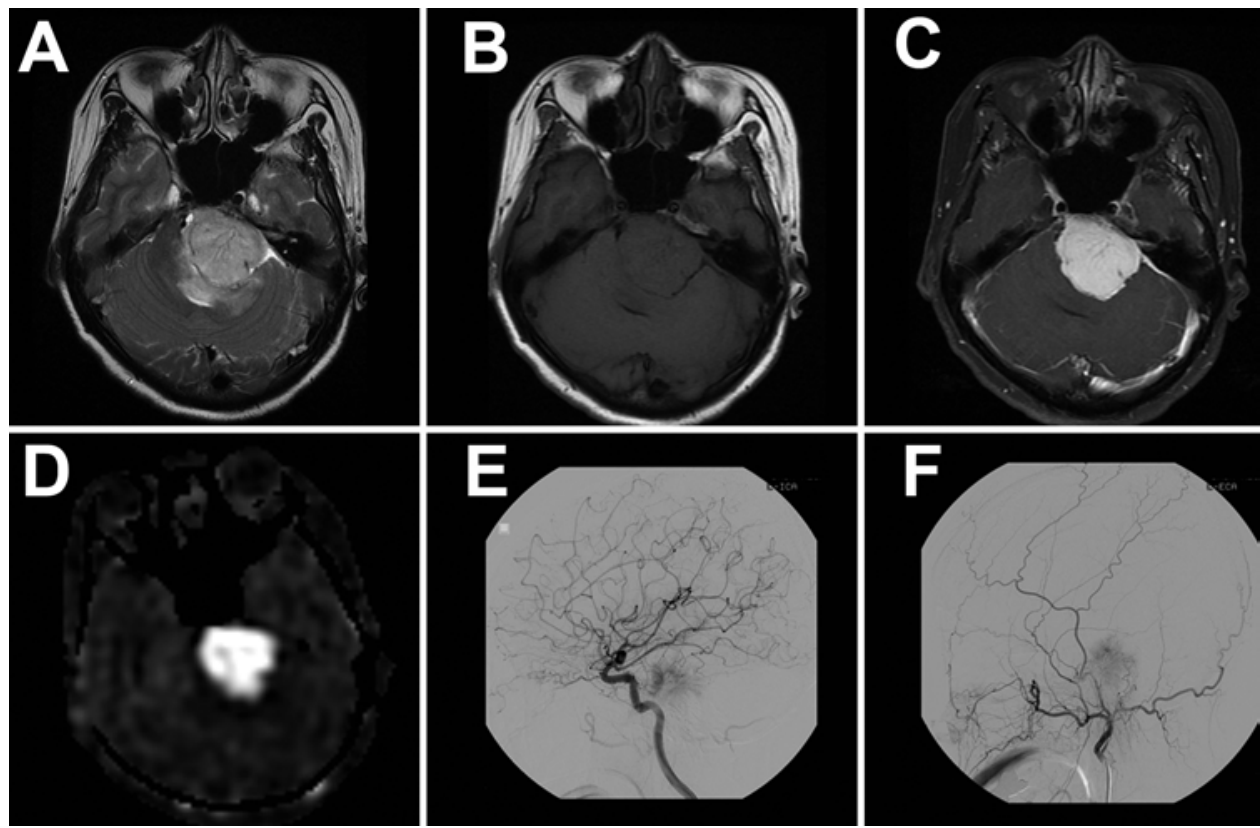

FIG. 4. High-vascularity meningioma in a 56-year-old woman. Axial T2- (A) and T1-weighted (B and $\mathbf{C}$ ) weighted images show a T2 iso- to hyperintense, strongly enhancing dural-based mass at the left cerebellopontine angle. Pseudocontinuous ASL-PWI (D) acquired at 1.5 T demonstrates a marked elevation of CBF within the mass ( $\mathrm{nCBF}=8.617)$. On selective DSAs of the ICA $(E)$ and ECA (F) (lateral projections), the mass was interpreted to have Grade 2 angiographic vascularity as a whole, based on the summation of grades noted on each DSA (ICA: Grade 1; ECA: Grade 1). The tumor was mainly fed by clival dural branches of the left ICA and petrous branches of the left middle meningeal artery. Preoperative embolization was not performed due to the small size of arterial feeders. 
preoperative embolization could be a safe option for selected meningiomas, with its degree being an independent predictor of decreased operative blood loss. ${ }^{13}$ The authors speculated that patients with high nCBF values from ASL maps would have high-vascularity meningiomas and thus be potential candidates for preoperative DSA and embolization. We found that the nCBF of the high-vascularity group, the majority (12 of 16 [75\%]) of whom underwent preoperative embolization, was significantly higher than that of the low-vascularity group; this finding was consistent with that of the previous study..$^{17}$ In the present study, diagnostic performance of $\mathrm{nCBF}$ in the differential diagnosis between the 2 groups was high, with an area under the ROC curve of 0.875 , a sensitivity of $69 \%$, and a specificity of $100 \%$ at the optimal cutoff value of 1.733 .

However, further analysis, according to whether preoperative embolization was performed, showed no significant difference in the nCBF between the group with embolization and the group with DSA only. The lack of a statistically significant difference was partly due to a few exceptional cases, in which embolization was not performed despite the high vascularity. The discrepancy can be attributed to the fact that several variables, such as the size, tortuosity, and origin of arterial feeders, need to be factored into decision making for embolization to reduce technical difficulty and procedure-related complications, which have been reported to be as high as $4.6 \% .{ }^{15}$ It is expected that incorporation of additional information on the vascular supply or anatomy of main arterial feeders, as provided by MR angiography and regional perfusion imaging, will help refine candidate selection in the future.

At the present time, we infer that ASL-PWI may predict angiographic vascularity, which in turn is a key determinant of the need for preoperative embolization; thus, it may aid in selecting potential candidates for preoperative DSA and embolization. Furthermore, in our study population, DSA was initially performed because surgeons, based on their reviews of MR images, considered that embolization may be needed and thus referred the patients to neurointerventionists. In some of the patients, however, embolization was not performed because the vascularity was shown to be low on DSA. Given the high specificity (100\%) for differentiating between the high- and low-vascularity groups at the optimal nCBF cutoff value, we speculate that the unnecessary DSA could have been avoided in the first place had the ASL findings been taken into consideration. In addition, the use of ASL-PWI as an initial screening tool would reduce the length of stay and cost for patients and lead to more economical use of hospital resources.

Our study had a few limitations. First, the degree of angiographic vascularity was visually assessed using a 4-point grading scale, rather than by means of objective measurement. The use of visual assessment, however, is more practical in the routine clinical setting. Furthermore, substantial interobserver agreement supports that it may be a good alternative. Second, ASL-PWI was performed using MR scanners with 2 different magnetic field strengths and ASL labeling methods. However, we attempted to minimize the influence of these differences by normalizing CBF values of the tumors to the contralateral normal side. Third, the sample size in the present study is small, and validation of the optimal nCBF cutoff value on ASL-PWI is needed in large case series. Fourth, embolization was done in a few patients with Grade 1 angiographic vascularity (low vascularity) due to the treating interventionist's preference or the relative ease of microcatheter navigation. If the study had been designed prospectively, we could have had our neurointerventionists perform embolization when it was definitely indicated, which would have strengthened the point that ASL-PWI may help avoid unnecessary invasive angiographic evaluation.

\section{Conclusions}

Arterial spin labeling perfusion-weighted imaging may provide a reliable and noninvasive means of predicting angiographic vascularity in meningiomas, which in turn is a key determinant of the need for preoperative embolization. In clinical practice, ASL-PWI may help select potential candidates for preoperative DSA and embolization and, at the same time, avoid unnecessary invasive angiographic evaluation.

\section{Acknowledgments}

This study was supported by a grant from the National Research Foundation of Korea (NRF-2013R1A1A2008332).

\section{References}

1. Alsop DC, Detre JA, Golay X, Günther M, Hendrikse J, Hernandez-Garcia L, et al: Recommended implementation of arterial spin-labeled perfusion MRI for clinical applications: A consensus of the ISMRM perfusion study group and the European consortium for ASL in dementia. Magn Reson Med, 2014

2. Borg A, Ekanayake J, Mair R, Smedley T, Brew S, Kitchen $\mathrm{N}$, et al: Preoperative particle and glue embolization of meningiomas: indications, results, and lessons learned from 117 consecutive patients. Neurosurgery 73 (2 Suppl Operative):ons244-ons252, 2013

3. Cebeci H, Aydin O, Ozturk-Isik E, Gumus C, Inecikli F, Bekar A, et al: Assessment of perfusion in glial tumors with arterial spin labeling; comparison with dynamic susceptibility contrast method. Eur J Radiol 83:1914-1919, 2014

4. Dawkins AA, Evans AL, Wattam J, Romanowski CA, Connolly DJ, Hodgson TJ, et al: Complications of cerebral angiography: a prospective analysis of 2,924 consecutive procedures. Neuroradiology 49:753-759, 2007

5. Detre JA, Leigh JS, Williams DS, Koretsky AP: Perfusion imaging. Magn Reson Med 23:37-45, 1992

6. Dixon WT, Du LN, Faul DD, Gado M, Rossnick S: Projection angiograms of blood labeled by adiabatic fast passage. Magn Reson Med 3:454-462, 1986

7. Dowd CF, Halbach VV, Higashida RT: Meningiomas: the role of preoperative angiography and embolization. Neurosurg Focus 15(1):E10, 2003

8. Järnum H, Steffensen EG, Knutsson L, Fründ ET, Simonsen $\mathrm{CW}$, Lundbye-Christensen S, et al: Perfusion MRI of brain tumours: a comparative study of pseudo-continuous arterial spin labelling and dynamic susceptibility contrast imaging. Neuroradiology 52:307-317, 2010

9. Kimura H, Takeuchi H, Koshimoto Y, Arishima H, Uematsu $\mathrm{H}$, Kawamura $\mathrm{Y}$, et al: Perfusion imaging of meningioma by using continuous arterial spin-labeling: comparison with dynamic susceptibility-weighted contrast-enhanced MR images and histopathologic features. AJNR Am J Neuroradiol 27:85-93, 2006 
10. Knutsson L, van Westen D, Petersen ET, Bloch KM, Holtås S, Ståhlberg F, et al: Absolute quantification of cerebral blood flow: correlation between dynamic susceptibility contrast MRI and model-free arterial spin labeling. Magn Reson Imaging 28:1-7, 2010

11. Koizumi S, Sakai N, Kawaji H, Takehara Y, Yamashita S, Sakahara H, et al: Pseudo-continuous arterial spin labeling reflects vascular density and differentiates angiomatous meningiomas from non-angiomatous meningiomas. J Neurooncol 121:549-556, 2015

12. Noguchi T, Yoshiura T, Hiwatashi A, Togao O, Yamashita K, Nagao E, et al: Perfusion imaging of brain tumors using arterial spin-labeling: correlation with histopathologic vascular density. AJNR Am J Neuroradiol 29:688-693, 2008

13. Raper DM, Starke RM, Henderson F Jr, Ding D, Simon S, Evans AJ, et al: Preoperative embolization of intracranial meningiomas: efficacy, technical considerations, and complications. AJNR Am J Neuroradiol 35:1798-1804, 2014

14. Schraml C, Müssig K, Martirosian P, Schwenzer NF, Claussen CD, Häring HU, et al: Autoimmune thyroid disease: arterial spin-labeling perfusion MR imaging. Radiology 253:435-442, 2009

15. Shah AH, Patel N, Raper DM, Bregy A, Ashour R, Elhammady MS, et al: The role of preoperative embolization for intracranial meningiomas. J Neurosurg 119:364-372, 2013

16. Thiex R, Norbash AM, Frerichs KU: The safety of dedicatedteam catheter-based diagnostic cerebral angiography in the era of advanced noninvasive imaging. AJNR Am J Neuroradiol 31:230-234, 2010

17. Toh $\mathrm{CH}$, Wei $\mathrm{KC}$, Chang CN, Peng YW, Ng SH, Wong HF, et al: Assessment of angiographic vascularity of meningiomas with dynamic susceptibility contrast-enhanced perfusionweighted imaging and diffusion tensor imaging. AJNR Am J Neuroradiol 35:263-269, 2014

18. van Westen D, Petersen ET, Wirestam R, Siemund R, Bloch KM, Ståhlberg F, et al: Correlation between arterial blood volume obtained by arterial spin labelling and cerebral blood volume in intracranial tumours. MAGMA 24:211-223, 2011

19. Warmuth C, Gunther M, Zimmer C: Quantification of blood flow in brain tumors: comparison of arterial spin labeling and dynamic susceptibility-weighted contrast-enhanced MR imaging. Radiology 228:523-532, 2003

20. Wiemels J, Wrensch M, Claus EB: Epidemiology and etiology of meningioma. J Neurooncol 99:307-314, 2010
21. Willinsky RA, Taylor SM, TerBrugge K, Farb RI, Tomlinson G, Montanera W: Neurologic complications of cerebral angiography: prospective analysis of 2,899 procedures and review of the literature. Radiology 227:522-528, 2003

22. Wolf RL, Wang J, Wang S, Melhem ER, O'Rourke DM, Judy $\mathrm{KD}$, et al: Grading of CNS neoplasms using continuous arterial spin labeled perfusion MR imaging at 3 Tesla. J Magn Reson Imaging 22:475-482, 2005

23. Wong AM, Yan FX, Liu HL: Comparison of three-dimensional pseudo-continuous arterial spin labeling perfusion imaging with gradient-echo and spin-echo dynamic susceptibility contrast MRI. J Magn Reson Imaging 39:427-433, 2014

24. Yoo RE, Choi SH, Cho HR, Kim TM, Lee SH, Park CK, et al: Tumor blood flow from arterial spin labeling perfusion MRI: a key parameter in distinguishing high-grade gliomas from primary cerebral lymphomas, and in predicting genetic biomarkers in high-grade gliomas. J Magn Reson Imaging 38:852-860, 2013

\section{Disclosures}

The authors report no conflict of interest concerning the materials or methods used in this study or the findings specified in this paper.

\section{Author Contributions}

Conception and design: Yun. Acquisition of data: Yun, Yoo. Analysis and interpretation of data: Yun, Yoo, Cho. Drafting the article: Yun, Yoo. Critically revising the article: Yun, Yoo, Rhim, KM Kang, Choi, JH Kim, JE Kim, HS Kang, Sohn, Park, Han. Reviewed submitted version of manuscript: all authors. Approved the final version of the manuscript on behalf of all authors: Yun. Statistical analysis: Yun, Yoo. Administrative/technical/material support: Yun. Study supervision: Yun.

\section{Correspondence}

Tae Jin Yun, Department of Radiology, Seoul National University Hospital, 101, Daehangno, Jongno-gu, Seoul 110-744, Korea.email: radiologyyun@gmail.com. 